\title{
An extended Li-Fraumeni kindred with gastric carcinoma and a codon 175 mutation in TP53
}

\author{
J M Varley, G McGown, M Thorncroft, K J Tricker, M D Teare, \\ M F Santibanez-Koref, R S Houlston, J Martin, J M Birch, D G R Evans
}

\begin{abstract}
We present an extended family with $\mathrm{Li}$ Fraumeni syndrome characterised by gastric and breast carcinoma, glioma, sarcoma, and leukaemia. This family showed strong evidence of linkage to TP53, and three of four tumours analysed showed loss of the wild type allele. A codon 175 missense mutation was identified in exon 5 in all available affected subjects. Counselling, screening, and issues surrounding presymptomatic testing are discussed.
\end{abstract}

( $($ Med Genet 1995;32:942-945)

It has been known for some time that common malignancies can cluster together in a fashion indicating that hereditary factors are involved. Furthermore the existence of site specific breast, ${ }^{1}$ colon, ${ }^{2}$ and ovarian ${ }^{3}$ cancer families showing autosomal dominant inheritance have long been described. Cancers may also segregate together, for example, breast and ovary ${ }^{4}$ and endometrium and colon. ${ }^{5}$ The latter group, hereditary non-poplyposis colorectal cancer, ${ }^{6}$ also includes cancer of the ovary, pancreas, and breast. The combination of childhood soft tissue sarcomas and early onset of breast cancer in mothers and other female relatives, together with adrenocortical tumours, brain tumours, osteosarcoma, and leukaemia occurring to excess, is also a recognised syndrome. ${ }^{7}$ This is known as the Li-Fraumeni syndrome (LFS) and was first described in $1969 .^{8}$ Germline mutations involving the TP53 gene have been shown to account for the majority, but not all, of classical LFS families. ${ }^{9-12}$ We describe an LFS family unusual for the presence of two gastric carcinomas and in which the structure of the family was sufficient to establish linkage

Received 25 May 1995 Revised version accepted fo publication 22 August 1995 to TP53. Subsequent DNA sequence analysis showed a missense mutation in codon 175 in exon 5 of TP53 in the family.

\section{Patients and methods}

The family (figure) was ascertained through the mother of IV.5 who had asked for counselling on the child's cancer risks. At about the same time, III. 5 also presented, worried about the recent death of his brother (III-2). Initially it was only possible to ascertain affected cases up to II 2 ; however, after two years of extensive research, the full extent of the tumours in this branch of the family was established (III.8, III.13, IV·6). Diagnoses of the tumours were confirmed through death certificates, hospital records, and pathology reports and these were further checked by using the relevant cancer registry. Confirmation of histology was possible in all cases except I.1 and II·3. The diagnosis and age at presentation and survival of each case is presented where known (figure, table). There were no dermatological or other phenotypic markers on examining affected subjects.

\section{Molecular studies}

Initially no living affected family member was identified, therefore tumour (paraffin block) material was obtained from three subjects who had died (III·2, III $\cdot 6$, and IV·2), enabling analysis of DNA from affected subjects. More recently, a blood sample from a living affected subject (III-3) has become available, plus tumour material from III.8 and blood from unaffected subjects (III 1 , III $\cdot 5$, III $\cdot 11$, III $\cdot 15$, and IV $\cdot 1$; all given for research purposes only). Normal cervix and CINII/III tumour material were obtained from IV 14 . DNA samples were therefore available from 11 family members, five affected and four at $50 \%$ risk.

Initial studies of the TP53 gene involved mutation detection screening of DNA from the tumour of IV.2 using the HOT technique, which failed to detect a mutation. ${ }^{11}$ A polymorphic dinucleotide microsatellite repeat mapping within the TP53 gene ${ }^{13}$ was used to analyse segregation of TP53 alleles through the family, and samples from III $\cdot 2$, III 5 , III 6 , III $\cdot 11$, III 13 , III $\cdot 15$, IV 1 , and IV $\cdot 2$ were studied. A shared TP53 allele was identified in III $\cdot 2$, III 6 , III $\cdot 13$ IV 1 , and IV 2 , all of whom were affected with the exception of IV 1 . The linkage data indicated that a mutation within or close to the TP53 gene could be the causative defect, and that IV' 1 was an unaffected carrier. Lod scores were calculated using penetrance

* Still living but in relapse.

Mean survival from diagnosis, where known, 11.2 months.

$\mathrm{NK}$
0
1
$\mathrm{NK}$
$<1$
12
1
41
8
33
$<1$
9
$12^{*}$




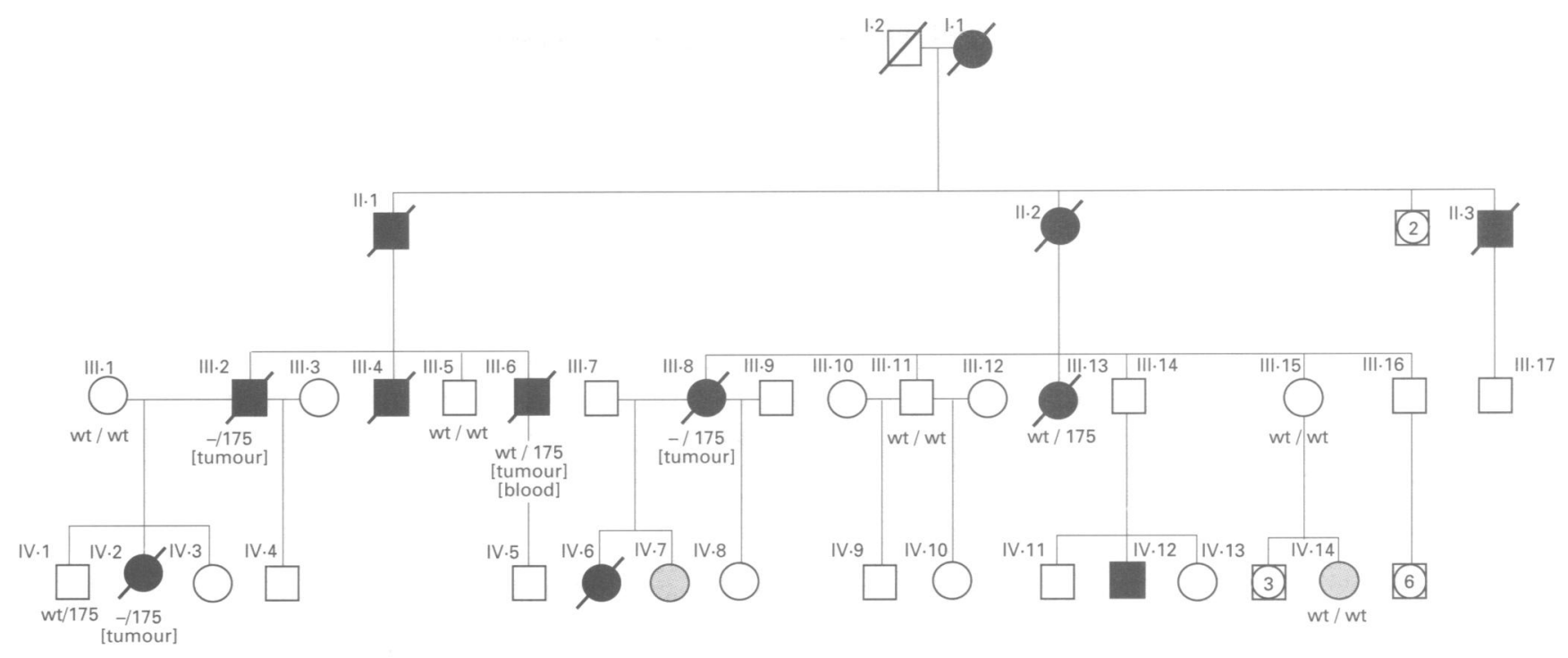

probabilities as described ${ }^{14}$ and were estimated at 1.24 $(\theta=0)$ without incorporating the loss of heterozygosity ( $\mathrm{LOH}$ ) data (see below) and $2 \cdot 17(\theta=0, \rho=0)$ including the LOH data. ${ }^{1516}$

When further DNA samples became available from affected and unaffected subjects, we sequenced the entire coding region in two subjects (III.8, III-13) using Sequenase dyeterminator chemistry and an ABI model 373 sequencer, and previously published primer sequences to analyse the entire gene exon by exon. ${ }^{11}$ An identical missense mutation was identified in exon 5 in DNA from blood from III. 13 and tumour from III $\cdot 8$. This mutation was within codon $175 \quad(\mathrm{CGC} \rightarrow \mathrm{CAC}$, Arg $\rightarrow$ His), and altered a recognition site for the endonuclease HhaI. Amplification of exon 5 followed by digestion with Hhal allowed resolution of wild type from mutant alleles and permitted us to analyse rapidly all the available DNA samples from the family. Direct sequencing of exon 5 was used to confirm the presence or absence of a mutation. Of the samples available, six showed a mutation (III $\cdot 2$, III $\cdot 6$, III $\cdot 8$, III 13 , IV 1 , and IV·2) confirming the linkage data. Five people did not have the mutation (III 11 , III $\cdot 5$, III 11 , III $\cdot 15$, and IV·14). III $\cdot 5$, III $\cdot 11$, and III 15 had previously been assessed at $50 \%$ risk, although linkage to TP53 had indicated that they were unaffected carriers.

DNA from four tumours were available; three showed loss of the wild type allele (III-2, III $\cdot 8$, and IV·2).

\section{Counselling issues}

Involvement of this family in research to identify the causative gene has stimulated one of two responses. Of the people initially at $50 \%$ risk, III·5, III 11 , III $\cdot 15, \mathrm{IV} \cdot 1, \mathrm{IV} \cdot 3, \mathrm{IV} \cdot 5$, and IV 8 have all been referred for formal genetic counselling. Standard genetic counselling was offered, including estimation of risk. However, IV. 7 and III. 14 have refused to take part even in the research aspects of the study. Even after the presentation of his son (IV-12) with an unusual leukaemia, III 4 continued to prefer not to become involved in the research or to have genetic counselling. Thus samples from IV.12 have not been available for study.

\section{Screening}

Subjects at risk have been offered open access to the genetics service. There is a very low threshold for extensive investigation of any abnormal symptom or sign. In addition, children are offered an annual ultrasound scan of the abdomen and pelvis, and a full blood count and film, while adults are offered a general physical examination. Breast screening in women involves an annual breast examination and ultrasound scan, but with a baseline mammogram at 25 years. One person (III.5) has opted for three yearly upper GI tract endoscopy.

\section{Discussion}

The family studied has early onset tumours in four generations, colon and endometrial cancer are absent, and the malignancies have been particularly severe. Two people developed a childhood sarcoma, which is classical of LFS, as is the presence of brain tumours in four subjects. An increase in the risk of breast cancer in mothers of children with osteosarcoma and chondrosarcoma has been reported, ${ }^{17}$ and is shown in this family. The presence of an adrenal cortical adenoma is also characteristic of LFS families, although most reports specify adrenal cortical carcinoma. The development of two gastric and one laryngeal cancer at young ages in this family is unusual for LFS. A report of tumours in 43 LFS families contained only four gastric carcinomas out of a total of 231 tumours. $^{9}$ Previous reports in which gastric tumours feature in the pedigree ${ }^{18}$ can be confusing, as the cancers occur in the branch of the family assumed not to be involved. However, it is clearly a component of some germline TP53 mutation families. A codon 282 mutation in exon 8 has been described in a family with four cases of gastric carcinoma, ${ }^{19}$ and in the present 
report we have shown the presence of the codon 175 mutation in the family, including in DNA from a patient with clear cell gastric adenocarcinoma (III-6). Three of the four tumours reported here have shown loss of the wild type allele, suggesting that the mutant TP53 is functioning as a tumour suppressor gene in this family.

The family reported here (family 117) has been part of a previous study in which it was described as one of the $50 \%$ of 12 classical LFS families in which a mutation in TP53 was not found. ${ }^{11}$ This may have been a consequence of the non-availability of DNA from a living affected subject at the time of the original study, and the use of tumour DNA for the mutation analysis. The adrenal cortical adenoma used in the original study appears to have lost the wild type allele, and it may therefore not have been possible to identify the missense mutation using the HOT technique, which detects mismatches between mutant and wild type sequences. Alternatively, the HOT technique may have been unable to detect this particular type of mutation. With the subsequent availability of other DNA samples from family members and the apparent linkage to TP53, we were prompted to sequence the entire coding region, leading to the identification of the causative mutation. We have previously noted that among LFS families the presence of an adrenal cortical carcinoma in a young child appears to confer a high chance of carrying a TP53 mutation in the germline. ${ }^{11}$ The finding of the codon 175 mutation in this family, which includes an infant with an adrenal cortical adenoma, adds weight to this observation.

Family 117 is one of the largest LFS families reported with 12 affected subjects and a further one obligate carrier (III-14). It is rare to have a family sufficiently large and with sufficient affected subjects on whom DNA is available to undertake linkage. While only $50 \%$ of classical LFS families have an identifiable reading frame mutation, it is thought that up to $85 \%$ involve TP53. ${ }^{20}$ This may be because of promoter sequence mutations or other mechanisms that may only be detected by allele specific expression studies. There are clearly LFS families in which coding mutations within TP53 have been excluded ${ }^{2122}$ and therefore a sizeable lod score would have to be generated to offer predictive testing based on linkage to TP53 alone. Using Easton's model ${ }^{23}$ for predictive testing for BRCA1 linkage in breast-ovary families, it would be necessary to generate a lod score in excess of 1.0 if mutation studies had not been carried out, and of 1.5 if they had (and no mutation found), to obtain $95 \%$ confidence with informative flanking markers. This would take into account that up to $40 \%$ of LFS families without a detectable coding TP53 coding sequence mutation were nevertheless associated with an inherited TP53 gene abnormality. If the LOH data were not incorporated into the linkage calculation, the lod score was $1 \cdot 24(\theta=0)$, although inclusion of $\mathrm{LOH}$ data raised this to $2 \cdot 17(\theta=0, \rho=0)$. Even in a large family such as this it is difficult to obtain material on enough relatives to achieve sufficient power to detect linkage. TP53 is a tumour suppressor gene, and so loss of the wild type allele in a tumour is further evidence for TP53 involvement. Thus, allele loss in an affected carrier can be treated as an additional meiosis. Inclusion of such LOH data is particularly suitable in $\mathrm{Li}$-Fraumeni families where many affected cases have multiple primary tumours, each of which can be treated as an independent event (meiosis). However, offering a linkage diagnosis could be problematical and is still based largely on assumptions. In the present family, we were uncomfortable offering linkage diagnosis even though there was considerable pressure for us to do so, but with the identification of the causative mutation, we have a robust diagnostic test.

Although five members of this family have already expressed a very strong interest in knowing their TP53 status, predictive testing must be offered with caution. The identification of carrier status would imply a near $90 \%$ risk of malignancy by 50 years of age ${ }^{11}$ which in this family has resulted in death within a year in the majority of affected people. There is an understandably deeply entrenched fear of cancer in this family and in the absence of proven benefit from intervention, even genetic counselling should not be forced upon family members. III 14 , who would be identified as a gene carrier in counselling, may cope better psychologically by using denial, even if he feels he carries the mutation. Experience with predictive testing in Huntington's families according to a standard protocol has shown a low uptake for testing. ${ }^{24}$ Use of a standard protocol for predictive testing in potential carriers of TP53 mutations has been recommended. ${ }^{26-28}$ It is likely that if there is careful counselling a similarly low uptake for testing will occur in Li-Fraumeni families. At present, while there is no evidence that screening confers a benefit to children with LFS, predictive testing in childhood as in Huntington's disease is probably inadvisable. ${ }^{2426-28}$ There are, however, a number of subjects who would at counselling be at $50 \%$ risk, but who would not carry the mutation. Benefits for these people may be considerable.

An accepted protocol for disease screening in LFS families has yet to be devised. The evidence from TP53 deficient mice for susceptibility to the effects of radiation ${ }^{29}$ means that mammography (or other radiological screening such as CT) should not be used as a regular tool. However, ultrasound is even less effective than mammography in identifying early malignant lesions in the breasts of young women. The risk of breast cancer in LFS would justify some form of screening from 20 years of age ( $30 \%$ of breast cancers occur at $<30$ years in TP53 mutation carriers ${ }^{11}$ ), and magnetic resonance imaging may well be the way forward. However, the range and diversity of site of the tumours in LFS mean that even a total body MRI may not be sufficient to have an effect on mortality. The short survival as a consequence of the aggressive nature of the tumours in this and other LFS families may mean that the lag time to symptoms is very 
small. Nonetheless, the pressure from family members to have at least some form of screening is often great.

1 Cady B. Familial bilateral cancer of the breast. Ann Surg 1970;172:264-72.

2 Kluge T. Familial cancer of the colon. Acta Chir Scand 1964;127:392-8.

3 Lewis ACW, Davison BC. Familial ovarian cancer. Lancet 1969;ii:235-7.

4 Lynch HT, Harris RE, Guirgis HA, Moloney K, Carmody LL, Lynch JF. Familial association of breast/ovarian carcinoma. Cancer 1978;41:1543-9.

5 Lynch HT, Shaw MW, Magnuson CW, Larsen AL, Krush AJ. Hereditary factors in cancer: study of two large midwestern kindreds. Ann Intern Med 1966;117:206-12.

6 Lynch HT, Lanspa SJ, Bonan BM, Smyth T, Watson P, Lynch JF. Hereditary non-polyposis colorectal cance Lynch syndromes I and II. Gastroenterol Clin N Am 1988; 174:679-715.

7 Birch JM. The Li Fraumeni cancer family syndrome. $f$ Pathol 1990;161:1-2.

8 Li FP, Fraumeni JF. Soft tissue sarcomas, breast cancer and other neoplasms. A familial syndrome? Ann Intern Med 1969;71:747-52.

9 Malkin D, Li FP, Strong LC, Fraumeni JF, et al. Germ line p53 mutations in a familial syndrome of breast cancer, sarcomas and other neoplasms. Science 1990;250:1233-8.

10 Santibanez-Koref MF, Birch JM, Hartley AL, et al. p53 germline mutations in Li-Fraumeni syndrome. Lancet germline mutation

11 Birch JM, Hartley $\mathrm{AL}$, Tricker KJ, et al. Prevalence and diversity of constitutional mutations in the $p 53$ gene amon $1298-304$

12 Frebourg T, Barbier N, Yan Y-X, et al. Germ-line p53 mutations in 15 families with $\mathrm{Li}$ Fraumeni syndrome. $A m$ f Hum Genet 1995;56:608-15.

13 Jones MH, Nakamura Y. Detection of loss of heterozygosity at the human TP53 locus using a dinucleotide repeat polymorphism. Genes Chrom Cancer 1992;5:89-90.

14 Lustbader ED, Williams RW, Bondy ML, Strom S, Strong L. Segregation analysis of cancer in families of childhood soft tissue sarcoma patients. Am f Hum Genet 1992;2: 344-56.
15 Rohde K, Teare MD, Schernek, Santibanez-Koref MF. A program using loss of constitutional heterozygosity data to ascertain the location of predisposing genes in cancer families. Hum Hered (in press).

16 Teare MD, Rhode K, Santibanez-Koref MF. The use of constitutional heterozygosity data to ascertain the location of predisposing genes in cancer families. $f$ Med Genet 1994;31:448-52.

17 Hartley AL, Birch JM, Marsden HB, Harris M. Breast cancer risk in mothers of children with osteosarcoma and chondrosarcoma. Br f Cancer 1986;54:819-23.

18 Pearson ADJ, Craft AW, Ratcliffe JM, Birch JM, Morris Jones P, Roberts DF. Two families with the Li-Fraumen cancer family syndrome. $\mathcal{F}$ Med Genet 1982;19:362-5.

19 Toguchida J, Yamaguchi T, Dayton SH, et al. Prevalence and spectrum of germ-line p 53 mutations among patients and spectrum of germ-line p53 mutations amon

20 Malkin D. Germline mutations to the p53 gene and genetic predisposition to cancer. Symposium on Genetic Prepredisposition to cancer. Symposium
disposition to Cancer SIOP 1994:24025.

21 Barnes DM, Hanby AM, Gillett CE, et al. Abnormal exparnes DM, Hanby AM, Gillett CE, et al. Abnormal ex-
pression of wild type p53 protein in normal cells of a cancer family patient. Lancet 1992;340:259-63.

22 Birch JM, Heighway J, Teare MD, et al. Linkage studies in a Li-Fraumeni family with increased expression of p53 protein but no germline mutation in p53. Br $\mathcal{f}$ Cancer 1994;70:1176-81.

23 Easton DF. Cancer Family Study Group guidelines for BRCA1 linkage predictive testing, 1993.

24 Ethical issues policy statement on Huntington's disease molecular genetic predictive test. $\mathcal{F}$ Med Genet 1990;27: 34-8.

25 Craufurd D, Kerzin-Storrar L, Dodge A, Harris R. Uptake of presymptomatic predicitive testing for Huntington's

$26 \mathrm{Li} \mathrm{FP,} \mathrm{Garber} \mathrm{JE,} \mathrm{Friend} \mathrm{SH,} \mathrm{et} \mathrm{al.} \mathrm{Recommendations}$ for predictive testing for germline p 53 mutations among cancer prone individuals. $\mathcal{F}$ Natl Cancer Inst 1992;84: 1156-60.

27 Birch JM. Li Fraumeni syndrome. Eur f Cancer 1994;13: 1935-41.

28 Eeles RA. Predictive testing for germline mutations in the p53 gene: are all the questions answered? Eur 7 Cancer 29: $1361-5$

29 Kemp CJ, Wheldon T, Balmain A. p53-deficient mice are extremely susceptible to radiation-induced tumorigenesis. Nature Genet 1994;8:66-9. 